\title{
Clinicopathological study of epithelial salivary gland tumours-a retrospective study
}

Jayawardana RADTM, Dias DK

\begin{abstract}
Introducton: Tumours of the salivary glands are infrequent, yet they represent an extensive variety of both benign and malignat tumours.
\end{abstract}

Objective: The aim of this study was to evaluate clinicopathological data to determine the distribution of epithelial salivary gland tumours(SGT) in patients who were diagnosed at OMFS unit, TH-Karapitiya, Sri Lanka from 2004 to 2014.

Material and methods: Total of 77 SGT were diagnosed in the period of 11 years were reviewed using biopsy reports.

Results: Out of 77 epithelial SGTs 35 were benign , 42 were malignat. Indicating a benign to malignat ratio of 1: 1.2. Malignat tumours were more common in minor salivary glands indicating a percentage of $59.52 \%$ of total malignat tumours.When considering minor SGT, $73.52 \%$ were malignat.

Conclusion: There was a higher occurrence of malignat tumours in minor salivary glands in this study.

Keywords : Salivary gland tumours, clinicopathological study

\section{Introduction}

Salivary gland tumours (SGTs) are a collection of heterogeneous lesions. These tumours are relatively rare comprising less than $1 \%$ of all tumours, and $3 \%-6$ $\%^{1,2}$ of tumours originating in the head and neck region. The estimated global incidence according to the World Health Organization ranges from 0.4 to 13.5 cases per 100,000 population yearly. ${ }^{1,3}$

Approximately $80 \%$ the tumours occur in parotid gland and $80 \%$ of them are benign. ${ }^{4}$ The remaining tumours occur in submandibular, sublingual and minor salivary glands. More than $50 \%$ of the submandibular and sublingual tumours are reported to be malignant. ${ }^{4}$ Whereas the majority of the minor SGTs are malignant. ${ }^{5}$ Though usually asymptomatic, clinical predictors such as rapid development, pain, ulceration, facial nerve

Oral and Maxillofacial Surgery Unit,Teaching Hospital Karapitiya,Galle,Sri Lanka. involvement and cervical lymphadenopathy may suggest malignancy. ${ }^{1}$ They show a remarkable diversity in histology and biological behavior, for example some benign tumours may recur or progress to malignant lesions. Therefore this may impose great difficulties in management.

Also it is found to have geographical variations and ethnic differences in the clinicopathological outline..$^{2,6}$ It is difficult to explain the epidemiological differences in their global distribution because the etiologic agent is not defined. ${ }^{7}$ Unlike other tumours in head and neck region alcohol and tobacco consumption are not found to be associated with SGTs ${ }^{1}$, and most of the SGTs are idiopathic. ${ }^{1}$

The aim of this retrospective study was to analyze the clinicopathological data to define the distribution of epithelial SGTs in patients who presented to OMFS Unit, Teaching Hospital, and Karapitiya, Sri Lanka from 2004 to 2014 .

\section{Materials and Methods}

Approval for this study was attained from the Ethical Review Committee, Faculty of Medicine, Ruhuna. Total of 82 epithelial SGTs diagnosed during the period of 11 years from 2004 to 2014 at OMFS Unit, Teaching Hospital , Karapitiya were reviewed . Age, gender, site distribution, and histological type (according to the 2005 WHO Classification of SGTs) were our variables. Data were collected retrospectively through biopsy reports. Reports with insufficient data were excluded from the study. (5 cases)

Statistical analysis of the results was performed with SPSS version 17.

\section{Results}

\section{General features}

A total of 77 epithelial SGTs were analyzed, 35 (45.45\%) were categorized as benign and 42 (54.5\%) as malignant. A benign-to-malignant ratio of 1:1.2 was indicated. Major glands were involved in 43 cases, of which 26 cases were benign and 17 were malignant. Minor glands were involved in 34 cases and 25 of them were malignant and only 9 were benign. 
(Table 1) Histopathological distribution of SGTs according to location

\begin{tabular}{|c|c|c|c|c|c|c|c|c|c|c|}
\hline & & Site & e les & & & & & & & क्व \\
\hline & hological Diagnosis & 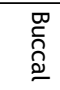 & 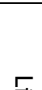 & $\stackrel{刃}{\circledR}$ & 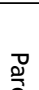 & $\stackrel{\check{E}}{\Xi}$ & 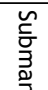 & $\frac{\check{E}}{g}$ & & \\
\hline 䀡. & Basal cell adenoma & 0 & 0 & 0 & 1 & 0 & 0 & 0 & 0 & 1 \\
\hline & Dutal oncocytoma & 0 & 2 & 0 & 0 & 0 & 0 & 0 & 0 & 2 \\
\hline & Myoepithelioma & 0 & 0 & 2 & 0 & 0 & 0 & 0 & 0 & 2 \\
\hline & Pleomorphic adenoma & 1 & 1 & 3 & 9 & 1 & 2 & 0 & 0 & 17 \\
\hline & Warthin's tumour & 0 & 0 & 0 & 13 & 0 & 0 & 0 & 0 & 13 \\
\hline$\frac{3}{0}$ & PLGA (polymorpho low grade adenocarcinoma) & 1 & 0 & 2 & 0 & 0 & 0 & 0 & 0 & 3 \\
\hline & Acinic cell CA & 1 & 0 & 1 & 2 & 1 & 1 & 0 & 0 & 6 \\
\hline & Adenoid cystic CA & 0 & 0 & 4 & 0 & 4 & 2 & 2 & 1 & 13 \\
\hline & Carcinosarcoma arising from Pleomorphic adenoma & 0 & 0 & 1 & 0 & 1 & 0 & 0 & 0 & 2 \\
\hline & Cystadenocarcinoma & 1 & 0 & 0 & 0 & 0 & 0 & 0 & 0 & 1 \\
\hline & Epimyoepithelial CA & 0 & 0 & 0 & 0 & 0 & 1 & 0 & 0 & 1 \\
\hline & Mucoepidermoid CA & 2 & 0 & 8 & 0 & 2 & 1 & 0 & 1 & 14 \\
\hline & Salivary duct CA & 0 & 0 & 0 & 1 & 0 & 1 & 0 & 0 & 2 \\
\hline To & & 6 & 3 & 21 & 26 & 9 & 8 & 2 & 2 & 77 \\
\hline
\end{tabular}

\section{Location}

Parotid gland tumours consisted of 26 (33.8\%) cases and 23 of them were benign. And $10.4 \%$ of all tumours occurred in submandibular gland while sublingual tumours accounted for $11.7 \%$.There were 34 minor SGTs.Palate was the most common location $(n=21)$ among the minor SGTs followed by buccal mucosa .(table 1$)$

(Table 2) Histopathological distribution of SGT according to type of gland and gender.

\begin{tabular}{|c|c|c|c|c|c|}
\hline \multirow{2}{*}{\multicolumn{2}{|c|}{ Histopathological Diagnosis }} & \multicolumn{2}{|c|}{ Type of the gland } & \multicolumn{2}{|c|}{ Gender } \\
\hline & & \multirow{2}{*}{$\begin{array}{l}\text { Minor } \\
0\end{array}$} & \multirow{2}{*}{$\begin{array}{l}\text { Major } \\
1\end{array}$} & \multirow{2}{*}{$\begin{array}{l}\text { Male } \\
0\end{array}$} & \multirow{2}{*}{$\begin{array}{l}\text { Female } \\
1\end{array}$} \\
\hline 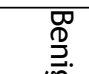 & Basal cell adenoma & & & & \\
\hline & Dutal oncocytoma & 2 & 0 & 1 & 1 \\
\hline & Myoepithelioma & 2 & 0 & 1 & 1 \\
\hline & Pleomorphic adenoma & 5 & 12 & 7 & 10 \\
\hline & Warthin's tumour & 0 & 13 & 13 & 0 \\
\hline \multirow{8}{*}{ 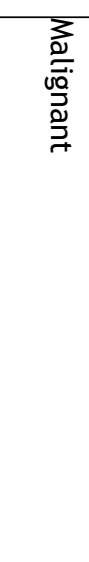 } & PLGA(polymorpho low grade adeno carcinoma) & 3 & 0 & 2 & 1 \\
\hline & Acinic cell CA & 2 & 4 & 2 & 4 \\
\hline & Adenoid cystic CA & 7 & 6 & 8 & 5 \\
\hline & Carcinosarcoma arising from Pleomorphic adenoma & 1 & 1 & 1 & 1 \\
\hline & Cystadenocarcinoma & 1 & 0 & 0 & 1 \\
\hline & Epimyoepithelial CA & 0 & 1 & 0 & 1 \\
\hline & Mucoepidermoid CA & 11 & 3 & 8 & 6 \\
\hline & Salivary duct CA & 0 & 2 & 1 & 1 \\
\hline \multicolumn{2}{|l|}{ Total } & 34 & 43 & 44 & 33 \\
\hline
\end{tabular}




\section{Age and gender}

SGTs were observed in the age ranging from 13years to 85 years. Overall mean age was 52.35 years. The affected patients were more commonly seen in the $6^{\text {th }}$ decade $(n=22)$. Malignant tumours occurred in older females (mean age of 52 years) compared to benign tumours (mean age of 46.5 years). But in males there was no significant age difference. In this study male to female ratio was 1:0.75. There was a large number of males having malignant and benign SGTs compared to females.

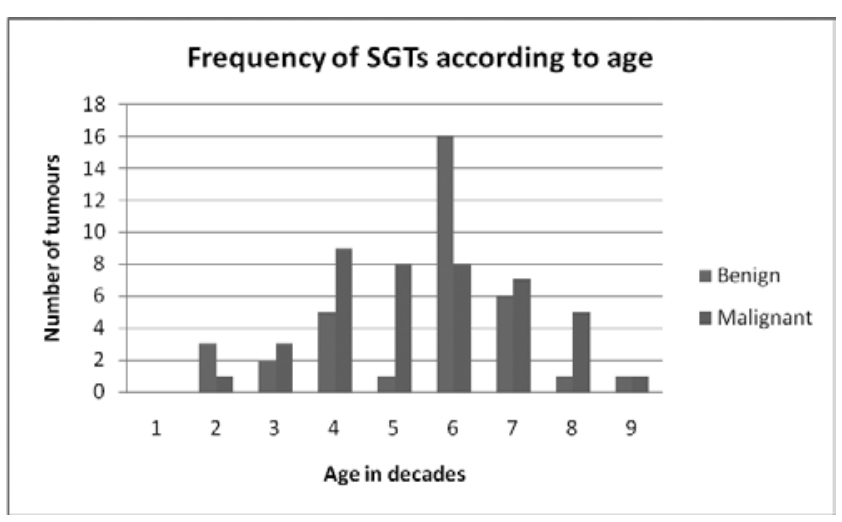

\section{Benign tumours}

Out of 35 benign SGTs pleomorphic adenoma and warthin's tumour comprised a percentage of $85 \%$ in our study.Pleomorphic adenoma was the most commonest. It represented $22 \%$ of all tumours and $48.57 \%$ of all benign tumours .

Warthin's tumour was the $2^{\text {nd }}$ common benign tumour ;seen only in parotid gland and only males were affected. It was the most commonest tumour of the parotid gland seen over pleomorphic adenoma.

\section{Malignant tumours}

Adenoid cystic and mucoepidermoid carcinomas were the two most commonest malignant SGTs in our study. Mucoepidermoid carcinoma was the second most commonest SGT accounting for $18.2 \%$ in both major and minor salivary glands. One third of malignant tumours were mucoepidermoid, with no involvement of parotid glands, commonly affecting the palate.Adenoid cystic carcinomas occurred both major and minor salivary glands, sparing the parotid gland.

\section{Discussion}

This retrospective study reviewed 77 SGTs of epithelial origin at our centre which is the largest referral centre for oral and maxillofacial tumours in southern Sri Lanka.

Most of the previous studies showed an equal frequency ${ }^{6}$ or higher percentages of benign tumours ${ }^{2,3,4}$ in SGTs. Ours revealed a benign to malignat ratio of $1: 1.2$, that is of almost equal rates. Also we found, minor SGTs to be predominantly malignant (73.5\%).This finding was seen in studies done in other countries as well. ${ }^{2,5}$ Yet studies in few regions in USA ${ }^{2}$ had shown a preponderance of benign tumours among minor salivary gland tumours which could have been ascribed to ethnic factors.

Conventionally the distribution of SGTs among sites has followed a ratio of 100:10:10:1 for parotid, submandibular, minor salivary glands, and sublingual tumours, respectively. ${ }^{3,9}$ As we observed relatively higher numbers of minor SGTs, our study did not conform to the above ratio. It may be due to geographical and environmental factors, for example studies from Uganda found no tumours involving the sublingual gland. ${ }^{3,9}$

As often reported we also found that most of the SGTs originated in the parotid gland. ${ }^{1,4}$ The commonest benign tumour of the parotid gland as reported in literature was pleomorphic adenoma . ${ }^{7}$ But in our study it was the Warthin's tumour. Similar to most of the other studies, the commonest malignant tumour was found to be mucoepidermoid carcinoma. ${ }^{6}$

Generally the remaining tumours arose in the submandibular gland trailed by minor salivary glands, ${ }^{4}$ but we found that minor salivary glands of the palate were affected more frequently than the submandibular gland. Our study also revealed that most tumours of the sublingual (89\%) and submandibular glands (75\%) were malignant, comparable to other Sri Lankan studies.

Most of the minor STGs showed a malignant propensity. In this present analysis $73.52 \%$ of minor SGTs were malignant and the commonest minor SGTs was mucoepidermoid carcinoma.

Pleomorphic adenoma was established to be the commonest benign tumour of all SGTs similar to almost all the published studies on SGTs. ${ }^{1,4,6,7}$ Though the literature reports a range of $40-72 \%$ of pleomorphic adenomas out of all SGTs ${ }^{6}$ in the present study we report only $22 \%$. This may be due to the controversies in SGTs classification which might have occurred throughout the years. For example some PLGA carcinomas may have been classified as pleomorphic adenomas in past. ${ }^{6}$ Pleomorphic adenoma was observed in higher prevalence among women (M:F ratio 0.7:1) approving with the findings in east China and Brazil, but contrasts with the predominance in men of Nigeria. ${ }^{3 .}$

Warthin's tumour was found only in the parotid gland and was the second commonest benign tumour. According to literature the usual incidence varies from $4 \%-13 \%{ }^{6}$ of all SGTs. But our study showed Warthin's tumours to be $37 \%$ of benign tumours and $16.8 \%$ of all SGTs.

Mucoepidermoid carcinoma was the commonest malignant tumour in the present study with a 
prevalence of $18.1 \%$, this was in accordance with most literature ${ }^{1,4,6}$ with a reported prevalence ranging from $2.1 \%$ to $17.2 \% .^{3}$ But a few studies conducted in Brazil and India showed adenoid cystic carcinoma to be the commonest among malignant tumours. ${ }^{2}$ This might have been due to some PLGA carcinomas been included along with adenoid cystic carcinomas and some high grade mucoepidermoid carcinomas been categorized as squamous cell carcinoma or unspecified adenocarcinoma. Otherwise it may be reflecting true ethnic and geographical variations. ${ }^{3}$ Mucoepidermoid carcinoma was also the most common minor SGT in this study in accordance with previous studies. ${ }^{5}$

Adenoid cystic carcinomas often present in parotid and submandibular glands. ${ }^{6}$ In contrast to previous studies our study found no adenoid cystic carcinomas in the parotid gland.

Most reports showed that SGTs were more common among women. ${ }^{3}$ However, in this study male to female ratio was 1:0.75 showing a slight male preponderance as seen in few previous studies. ${ }^{3,7}$ There was a male predominance in both malignant tumours $(52 \%)$ and benign(62\%). But literature shows that benign neoplasm occur more frequently in women than in men. ${ }^{6,4}$ Usually Warthin's tumour shows a strong male predilection, ${ }^{6}$ which is often attributed to the related habit of tobacco smoking. ${ }^{6}$ Our study too showed that Warthin's tumour was only seen in male individuals complying with Tilakaratne et al study done on Sri Lankan population. It might be due to the effect of low numbers of female smokers in Sri Lanka.

SGTs were observed in the ages ranging from 13 to 85.The mean ages of the affected patients were more or less similar for benign and malignant SGTs(50.2 and 51.74) agreeing with previous researches done on Sri Lankan population. But studies on populations of Brazil ${ }^{4}$ and Uganda ${ }^{9}$ showed a higher difference in mean ages. In the present study the peak incidence of benign tumours ,malignant tumours, benign and malignant tumours taken together were in the sixth decade. ${ }^{3}$ Benign tumours in females of our study tend to occur at younger mean ages(46.5yrs) compared to malignant (52 yrs). On the other hand males presented with malignant tumours at a slightly younger mean age( $51 \mathrm{yrs})$ in contrast to benign tumours ( $52.3 \mathrm{yrs}$ ). This finding differs with previous researches done on Sri Lankan population which had revealed an occurrence of malignant tumours in younger females and older men.

In summary, minor SGTs in this present study are characterized by a higher percentage of malignancy. Further studies should be designed to highlight the possible geographical and population-specific characteristics of these tumours.

\section{References}

1. Kalburg JV, Kalburg V, Latti B, Kini Y. Salivary Gland Tumors: Clinicopathologic analysis of 73 cases. J Cranio Max Dis 2014;3:111-5.

2. Jaafari-Ashkavandi Z, Ashraf MJ, Moshaverinia M (2013). Salivary gland tumors: a clinicopathologic study of 366 cases in Southern Iran. Asian Pac J Cancer Prev 2013; 14: 27-30.

3. Wang, Xiao-dong et al. Tumours of the salivary glands in northeastern China: a retrospective study of 2508 patients. British Journal of Oral and Maxillofacial Surgery, Volume 53, Issue 2, 132 - 137

4. Morais Mde L, Azevedo PR, Carvalho $\mathrm{CH}$, Medeiros L, Lajus T, Costa Ade L.Clinicopathological study of salivary gland tumors:an assessment of 303 patients. Cad Saude Publica 2011;27(5):1035-1040.

5. Strick MJ, Kelly C, Soames JV, McLean NR. Malignant tumours of the minor salivary glands-a 20 year review. British Journal of Plastic Surgery. 2004;57(7):624-631

6. Tilakaratne WM, Jayasooriya PR, Tennakoon TMPB,Saku T. Epithelial salivary tumours in Sri Lanka: a retrospective study of 713 cases. Oral Surg Oral Med Oral Pathol Oral Radiol Endod 2009; 108:90-8.

7. Subhashraj Krishnaraj. Salivary gland tumours: a single institution experience in India. Journal of Oral and Maxillofacial Surgery, 2008. Vol 46:635-638

8. Wang XD, Meng LJ, Hou TT, et al.Frequency and distribution pattern of minor salivary gland tumours in a northeastern Chinese population: a retrospective study of 485 patients. J Oral Maxillofac Surg. 2015; 73(1):81-91

9. EddaA.M Vuhahula.salivary gland tumours in uganda:clinical pathological study.African health sciences 2004;4(1):15-23 